\title{
Stability and Optimality in Matching Problems with Weighted Preferences
}

\author{
Maria Silvia Pini ${ }^{1}$, Francesca Rossi ${ }^{1}$, Kristen Brent Venable $^{1}$ and Toby Walsh ${ }^{2}$ \\ 1: Department of Pure and Applied Mathematics, University of Padova, Italy \\ \{mpini,frossi,kvenable\}@math.unipd.it \\ 2: NICTA and UNSW, Sydney, Australia \\ Toby.Walsh@nicta.com.au
}

\begin{abstract}
The stable marriage problem is a well-known problem of matching men to women so that no man and woman, who are not married to each other, both prefer each other. Such a problem has a wide variety of practical applications, ranging from matching resident doctors to hospitals, to matching students to schools or more generally to any two-sided market. In the classical stable marriage problem, both men and women express a strict preference order over the members of the other sex, in a qualitative way. Here we consider stable marriage problems with weighted preferences: each man (resp., woman) provides a score for each woman (resp., man). Such problems are more expressive than the classical stable marriage problems. Moreover, in some real-life situations it is more natural to express scores (to model, for example, profits or costs) rather than a qualitative preference ordering. In this context, we define new notions of stability and optimality, and we provide algorithms to find marriages which are stable and/or optimal according to these notions. While expressivity greatly increases by adopting weighted preferences, we show that in most cases the desired solutions can be found by adapting existing algorithms for the classical stable marriage problem. We also investigate manipulation issues in our framework. More precisely, we adapt the classical notion of manipulation to our context and we study if the procedures which return the new kinds of stable marriages are manipulable.
\end{abstract}

Keywords: stable marriages, weighted preferences

The stable marriage problem (SM) [9] is a well-known problem of matching the elements of two sets. It is called the stable marriage problem since the standard formulation is in terms of men and women, and the matching is interpreted in terms of a set of marriages. Given $n$ men and $n$ women, where each person expresses a strict ordering over the members of the opposite sex, the problem is to match the men to the women so that there are no two people of opposite sex who would both rather be matched with each other than their current partners. If there are no such people, all the marriages are said to be stable. In [4] Gale and Shapley proved that it is always possible to find a matching that makes all marriages stable, and provided a polynomial time algorithm which can be 
used to find one of two extreme stable marriages, the so-called male-optimal or female-optimal solutions. The Gale-Shapley algorithm has been used in many real-life scenarios [20], such as in matching hospitals to resident doctors [19], medical students to hospitals, sailors to ships [13], primary school students to secondary schools [21], as well as in market trading.

In the classical stable marriage problem, both men and women express a strict preference order over the members of the other sex in a qualitative way. Here we consider stable marriage problems with weighted preferences. In such problems each man (resp., woman) provides a score for each woman (resp., man). Stable marriage problems with weighted preferences are interesting since they are more expressive than the classical stable marriage problems, since in classical stable marriage problem a man (resp., a woman) cannot express how much he (resp., she) prefers a certain woman (resp., man). Moreover, they are useful in some real-life situations where it is more natural to express scores, that can model notions such as profit or cost, rather than a qualitative preference ordering. In this context, we define new notions of stability and optimality, we compare such notions with the classical ones, and we show algorithms to find marriages which are stable and/or optimal according to these notions. While expressivity increases by adopting weighted preferences, we show that in most cases the desired solutions can be found by adapting existing algorithms for the classical stable marriage problem. We also investigate manipulation issues in our framework. More precisely, we adapt the classical notion of manipulation to our context and we show that in some cases the procedures which return the new kinds of stable marriage are manipulable.

Stable marriage problems with weighted preferences have been studied also in $[8,12]$. However, they solve these problems by looking at the stable marriages that maximize the sum of the weights of the married pairs, where the weights depend on the specific criteria used to find an optimal solution, that can be minimum regret criterion [8], the egalitarian criterion [12] or the Lex criteria [12]. Therefore, they consider as stable the same marriages that are stable when we don't consider the weights. We instead use the weights to define new notions of stability that may lead to stable marriages that are different from the classical case. They may rely on the difference of weights that a person gives to two different people of the other sex, or by the strength of the link of the pairs (man,woman), i.e., how much a person of the pair wants to be married with the other person of the pair. The classical definition of stability for stable marriage problems with weighted preferences has been considered also in [2] that has used a semiring-based soft constraint approach [3] to model and solve these problems.

The paper is organized as follows. In Section 1 we give the basic notions of classical stable marriage problems, stable marriage problems with partially ordered preferences and stable marriage problems with weighted preferences (SMWs). In Section 2 we introduce a new notion of stability, called $\alpha$-stability for SMWs, which depends on the difference of scores that every person gives to two different people of the other sex, and we compare it with the classical notion of stability. Moreover, we give a new notion of optimality, called lex-optimality, 
to discriminate among the new stable marriages, which depends on a voting rule. We show that there is a unique optimal stable marriage and we give an algorithm to find it. In Section 3 we introduce other notions of stability for SMWs that are based on the strength of the link of the pairs (man,woman), we compare them with the classical stability notion, and we show how to find marriages that are stable according to these notions with the highest global link. In Section 4 we analyze manipulation issues in our context. In Section 5 we summarize the results contained in the paper, and we give some hints for future work.

A preliminary version of this paper has been presented in [16].

\section{Background}

We now give some basic notions on classical stable marriage problems, stable marriage problems with partial orders, and stable marriage problems with weighted preferences.

\subsection{Stable marriage problems}

A stable marriage problem (SM) [9] of size $n$ is the problem of finding a stable marriage between $n$ men and $n$ women. Such men and women each have a preference ordering over the members of the other sex. A marriage is a one-toone correspondence between men and women. Given a marriage $M$, a man $m$, and a woman $w$, the pair $(m, w)$ is a blocking pair for $M$ if $m$ prefers $w$ to his partner in $M$ and $w$ prefers $m$ to her partner in $M$. A marriage is said to be stable if it does not contain blocking pairs.

The sequence of all preference orderings of men and women is usually called a profile. In the case of classical stable marriage problem (SM), a profile is a sequence of strict total orders.

Given a SM $P$, there may be many stable marriages for $P$. However, it is interesting to know that there is always at least one stable marriage.

Given an SM $P$, a feasible partner for a man $m$ (resp., a woman $w$ ) is a woman $w$ (resp., a man $m$ ) such that there is a stable marriage for $P$ where $m$ and $w$ are married.

The set of all stable marriages for an SM forms a lattice, where a stable marriage $M_{1}$ dominates another stable marriage $M_{2}$ if men are happier (that is, are married to more or equally preferred women) in $M_{1}$ w.r.t. $M_{2}$. The top of this lattice is the stable marriage where men are most satisfied, and it is usually called the male-optimal stable marriage. Conversely, the bottom is the stable marriage where men's preferences are least satisfied (and women are happiest, so it is usually called the female-optimal stable marriage). Thus, a stable marriage is male-optimal iff every man is paired with his highest ranked feasible partner.

The Gale-Shapley (GS) algorithm [4] is a well-known algorithm to solve the SM problem. At the start of the algorithm, each person is free and becomes engaged during the execution of the algorithm. Once a woman is engaged, she never becomes free again (although to whom she is engaged may change), but 
men can alternate between being free and being engaged. The following step is iterated until all men are engaged: choose a free man $m$, and let $m$ propose to the most preferred woman $w$ on his preference list, such that $w$ has not already rejected $m$. If $w$ is free, then $w$ and $m$ become engaged. If $w$ is engaged to man $m^{\prime}$, then she rejects the man $\left(m\right.$ or $\left.m^{\prime}\right)$ that she least prefers, and becomes, or remains, engaged to the other man. The rejected man becomes, or remains, free. When all men are engaged, the engaged pairs form the male optimal stable matching. It is female optimal, of course, if the roles of male and female participants in the algorithm were interchanged.

This algorithm needs a number of steps that, in the worst case, is quadratic in $n$ (that is, the number of men), and it guarantees that, if the number of men and women coincide, and all participants express a strict order over all the members of the other group, everyone gets married, and the returned matching is stable.

Example 1. Assume $n=2$. Let $\left\{w_{1}, w_{2}\right\}$ and $\left\{m_{1}, m_{2}\right\}$ be respectively the set of women and men. The following sequence of strict total orders defines a profile:

$$
\begin{aligned}
& -m_{1}: w_{1}>w_{2} \text { (i.e., man } m_{1} \text { prefers woman } w_{1} \text { to woman } w_{2} \text { ), } \\
& -m_{2}: w_{1}>w_{2}, \\
& -w_{1}: m_{2}>m_{1}, \\
& -w_{2}: m_{1}>m_{2} .
\end{aligned}
$$

For this profile, the male-optimal solution is $\left\{\left(m_{1}, w_{2}\right),\left(m_{2}, w_{1}\right)\right\}$. For this specific profile the female-optimal stable marriage coincides with the male-optimal one.

\subsection{Stable marriage problems with partially ordered preferences}

In SMs, each preference ordering is a strict total order over the members of the other sex. More general notions of SMs allow preference orderings to be partial $[14,11,10,7,6]$. This allows for the modelling of both indifference (via ties) and incomparability (via absence of ordering) between members of the other sex. In this context, a stable marriage problem is defined by a sequence of $2 n$ partial orders, $n$ over the men and $n$ over the women. We will denote with SMP a stable marriage problem with such partially ordered preferences.

Given an SMP, we will sometimes use the notion of a linearization of such a problem, which is obtained by linearizing the preference orderings of the profile in a way that is compatible with the given partial orders.

A marriage $M$ for an SMP is said to be weakly-stable if it does not contain blocking pairs. Given a man $m$ and a woman $w$, the pair $(m, w)$ is a blocking pair if $m$ and $w$ are not married to each other in $M$ and each one strictly prefers the other to his/her current partner.

A weakly stable marriage $M$ dominates a weakly stable marriage $M^{\prime}$ iff for every man $m, M(m) \geq M^{\prime}(m)$ or $M(m) \bowtie M^{\prime}(m)$ ( $\bowtie$ means incomparable) and there is a man $m^{\prime}$ s.t. $M\left(m^{\prime}\right)>M^{\prime}\left(m^{\prime}\right)$. Notice that there may be more than one undominated weakly stable marriage for an SMP. 
Example 2. Let $\left\{w_{1}, w_{2}\right\}$ and $\left\{m_{1}, m_{2}\right\}$ be respectively the set of women and men. An instance of an SMP is the following:

$$
\begin{aligned}
& -m_{1}: w_{1}>\bowtie w_{2}, \\
& -m_{2}: w_{1}>w_{2}, \\
& -w_{1}: m_{1} \bowtie m_{2}, \\
& -w_{2}: m_{1}>m_{2} .
\end{aligned}
$$

For this instance, both $M_{1}=\left\{\left(m_{1}, w_{2}\right),\left(m_{2}, w_{1}\right)\right\}$ and $M_{2}=\left\{\left(m_{1}, w_{1}\right),\left(m_{2}, w_{2}\right)\right\}$ are weakly stable marriages and $M_{1}$ dominates $M_{2}$.

\subsection{Stable marriage problems with weighted preferences}

In classical stable marriage problems, men and women express only qualitative preferences over the members of the other sex. For every pair of women (resp., men), every man (resp., woman) states only that he (resp., she) prefers a woman (resp., a man) more than another one. However, he (resp., she) cannot express how much he (resp., she) prefers such a woman (resp., a man). This is nonetheless possible in stable marriage problems with weighted preferences.

A stable marriage problem with weighted preferences (SMW) [12] is a classical SM where every man/woman gives also a numerical preference value for every member of the other sex, that represents how much he/she prefers such a person. Such preference values are natural numbers and higher preference values denote a more preferred item. Given a man $m$ and a woman $w$, the preference value for man $m$ (resp., woman $w$ ) of woman $w$ (resp., man $m$ ) will be denoted by $p(m, w)$ (resp., $p(w, m))$.

Example 3. Let $\left\{w_{1}, w_{2}\right\}$ and $\left\{m_{1}, m_{2}\right\}$ be respectively the set of women and men. An instance of an SMW is the following:

$-m_{1}: w_{1}^{[9]}>w_{2}^{[1]}$ (i.e., man $m_{1}$ prefers woman $w_{1}$ to woman $w_{2}$, and he prefers $w_{1}$ with value 9 and $w_{2}$ with value 1 ),

$-m_{2}: w_{1}^{[3]}>w_{2}^{[2]}$,

$-w_{1}: m_{2}^{[2]}>m_{1}^{[1]}$

$-w_{2}: m_{1}^{[3]}>m_{2}^{[1]}$.

The numbers written into the round brackets identify the preference values.

In [12] they consider stable marriage problems with weighted preferences by looking at the stable marriage that maximizes the sum of the preference values. Therefore, they use the classical definition of stability and they use preference values only when they have to look for the optimal solution. We want, instead, to use preference values also to define new notions of stability and optimality.

We will introduce new notions of stability and optimality that are based on the weighted preferences expressed by the agents and we will show how to find them by adapting the classical Gale-Shapley algorithm [4] for SMs described in Section 1. 


\section{$2 \quad \alpha$-stability}

A simple generalization of the classical notion of stability requires that there are not two people that prefer with at least degree $\alpha$ (where $\alpha$ is a natural number) to be married to each other rather than to their current partners.

Definition 1 ( $\alpha$-stability). Let us consider a natural number $\alpha$ with $\alpha \geq 1$. Given a marriage $M$, a man $m$, and a woman $w$, the pair $(m, w)$ is an $\alpha$-blocking pair for $M$ if the following conditions hold:

- $m$ prefers $w$ to his partner in $M$, say $w^{\prime}$, by at least $\alpha$ (i.e., $p(m, w)-$ $\left.p\left(m, w^{\prime}\right) \geq \alpha\right)$,

- $w$ prefers $m$ to her partner in $M$, say $m^{\prime}$, by at least $\alpha$ (i.e., $p(w, m)-$ $\left.p\left(w, m^{\prime}\right) \geq \alpha\right)$.

A marriage is $\alpha$-stable if it does not contain $\alpha$-blocking pairs. A man $m$ (resp., woman $w$ ) is $\alpha$-feasible for woman $w$ (resp., man $m$ ) if $m$ is married with $w$ in some $\alpha$-stable marriage.

\subsection{Relations with classical stability notions}

Given an SMW $P$, let us denote with $c(P)$, the classical SM problem obtained from $P$ by considering only the preference orderings induced by the preference values of $P$.

Example 4. Let us consider the SMW, $P$, shown in Example 3. The stable marriage problem $c(P)$ is shown in Example 1.

If $\alpha$ is equal to 1 , then the $\alpha$-stable marriages of $P$ coincide with the stable marriages of $c(P)$. However, in general, $\alpha$-stability allows us to have more marriages that are stable according to this definition, since we have a more relaxed notion of blocking pair. In fact, a pair $(m, w)$ is an $\alpha$-blocking if both $m$ and $w$ prefer each other to their current partner by at least $\alpha$ and thus pairs $\left(m^{\prime}, w^{\prime}\right)$ where $m^{\prime}$ and $w^{\prime}$ prefer each other to their current partner of less than $\alpha$ are not considered $\alpha$-blocking pairs.

The fact that $\alpha$-stability leads to a larger number of stable marriages w.r.t. the classical case is important to allow new stable marriages where some men, for example the most popular ones or the least popular ones, may be married with partners better than all the feasible ones according to the classical notion of stability.

Given an SMW $P$, let us denote with $I_{\alpha}(P)$ the set of the $\alpha$-stable marriages of $P$ and with $I(c(P))$ the set of the stable marriages of $c(P)$. We have the following results.

Proposition 1. Given an $S M W P$, and a natural number $\alpha$ with $\alpha \geq 1$,

- if $\alpha=1, I_{\alpha}(P)=I(c(P))$;

- if $\alpha>1, I_{\alpha}(P) \supseteq I(c(P))$. 
Given an SMP $P$, the set of $\alpha$-stable marriages of $P$ contains the set of stable marriages of $c(P)$, since the $\alpha$-blocking pairs of $P$ are a subset of the blocking pairs of $c(P)$.

Let us denote with $\alpha(P)$ the stable marriage with incomparable pairs obtained from an SMW $P$ by setting as incomparable every pair of people that don't differ for at least $\alpha$, and with $I_{w}(\alpha(P))$ the set of the weakly stable marriages of $\alpha(P)$. It is possible to show that the set of the weakly stable marriages of $\alpha(P)$ coincides with the set of the $\alpha$-stable marriages of $P$.

Theorem 1. Given an $S M W P, I_{\alpha}(P)=I_{w}(\alpha(P))$.

Proof. We first show that $I_{\alpha}(P) \subseteq I_{w}(\alpha(P))$. Assume that a marriage $M \notin$ $I_{w}(\alpha(P))$, we now show that $M \notin I_{\alpha}(P)$. If $M \notin I_{w}(\alpha(P))$, then there is a pair (man,woman), say $(m, w)$, in $\alpha(P)$ such that $m$ prefers $w$ to his partner in $M$, say $w^{\prime}$, and $w$ prefers $m$ to her partner in $M$, say $m^{\prime}$. By definition of $\alpha(P)$, this means that $m$ prefers $w$ to $w^{\prime}$ by at least degree $\alpha$ and $w$ prefers $m$ to $m^{\prime}$ by at least degree $\alpha$ in $P$, and so $M \notin I_{\alpha}(P)$. Similarly, we can show that $I_{\alpha}(P) \supseteq I_{w}(\alpha(P))$. In fact, if $M \notin I_{\alpha}(P)$, then there is a pair (man,woman), say $(m, w)$, in $P$ such that $m$ prefers $w$ to $w^{\prime}$ by at least degree $\alpha$ and $w$ prefers $m$ to $m^{\prime}$ by at least degree $\alpha$. By definition of $\alpha(P)$, this means that $m$ prefers $w$ to $w^{\prime}$ and $w$ prefers $m$ to $m^{\prime}$ in $\alpha(P)$ and so $M \notin I_{w}(\alpha(P))$, i.e., $M$ is not a weakly stable marriage for $\alpha(P)$.

This means that, given an SMW $P$, every algorithm that is able to find a weakly stable marriage for $\alpha(P)$ provides an $\alpha$-stable marriage for $P$.

Example 5. Assume that $\alpha$ is 2. Let us consider the following instance of an SMW, say $P$.

$$
\begin{aligned}
& -m_{1}: w_{1}^{[3]}>w_{2}^{[2]} \\
& -m_{2}: w_{1}^{[4]}>w_{2}^{[2]}, \\
& -w_{1}: m_{1}^{[8]}>m_{2}^{[5]}, \\
& -w_{2}: m_{1}^{[3]}>m_{2}^{[1]} .
\end{aligned}
$$

The SMP $\alpha(P)$ is the following:

$$
\begin{aligned}
& -m_{1}: w_{1} \bowtie w_{2}, \\
& -m_{2}: w_{1}>w_{2}, \\
& -w_{1}: m_{1}>m_{2}, \\
& -w_{2}: m_{1}>m_{2} .
\end{aligned}
$$

The set of the $\alpha$-stable marriages of $P$, that coincides with the set of the weakly stable marriages of $\alpha(P)$, by Theorem 1, contains the following marriages: $M_{1}=$ $\left\{\left(m_{1}, w_{1}\right),\left(m_{2}, w_{2}\right)\right\}$ and $M_{2}=\left\{\left(m_{1}, w_{2}\right),\left(m_{2}, w_{1}\right)\right\}$.

On the other hand, not all stable marriage problems with partially ordered preferences can be expressed as stable marriage problems with weighted preferences such that the stable marriages in the two problems coincide. More precisely, 
given any SMP problem $P$, we would like to be able to generate a corresponding SMW problem $P^{\prime}$ and a value $\alpha$ such that, in $P^{\prime}$, the weights of elements ordered in $P$ differ more than $\alpha$, while those of elements that are incomparable in $P$ differ less than $\alpha$. Consider for example the case of a partial order over six elements, defined as follows: $x_{1}>x_{2}>x_{3}>x_{4}>x_{5}$ and $x_{1}>y>x_{5}$. Then there is no way to choose a value $\alpha$ and a linearization of the partial order such that the weights of $x_{i}$ and $x_{j}$ differ for at least $\alpha$, for any $\mathrm{i}, \mathrm{j}$ between 1 and 5 , while at the same time the weight of $y$ and each of the $x_{i}$ 's differ for less than $\alpha$.

\subsection{Dominance and lex-male-optimality}

We recall that in SMPs a weakly-stable marriage dominates another weaklystable marriage if men are happier (or equally happy) and there is at least a man that is strictly happier. The same holds for $\alpha$-stable marriages. As in SMPs there may be more than one undominated weakly-stable marriage, in SMWs there may be more than one undominated $\alpha$-stable marriage.

Definition 2 (dominance). Given two $\alpha$-stable marriages, say $M$ and $M^{\prime}$, $M$ dominates $M^{\prime}$ if every man is married in $M$ to more or equally preferred woman than in $M^{\prime}$ and there is at least one man in $M$ married to a more preferred woman than in $M^{\prime}$.

Example 6. Let us consider the SMW shown in Example 5. We recall that $\alpha$ is 2 and that the $\alpha$-stable marriages of this problem are $M_{1}=\left\{\left(m_{1}, w_{1}\right),\left(m_{2}, w_{2}\right)\right\}$ and $M_{2}=\left\{\left(m_{1}, w_{2}\right),\left(m_{2}, w_{1}\right)\right\}$. It is possible to see that:

- $M_{2}$ does not dominate $M_{1}$ since, for $m_{1}, M_{1}\left(m_{1}\right)>M_{2}\left(m_{1}\right)$ and

- $M_{1}$ does not dominate $M_{2}$ since, for $m_{2}, M_{2}\left(m_{2}\right)>M_{1}\left(m_{2}\right)$.

We now discriminate among the $\alpha$-stable marriages of an SMW, by considering the preference values given by women and men to order pairs that differ for less than $\alpha$.

In this paper we will consider a marriage optimal when the most popular men are as happy as possible and they are married with their most popular best $\alpha$-feasible women. However, we could also consider a marriage optimal when the least popular men are as happy as possible and they are married with their most popular best $\alpha$-feasible women.

To compute a strict ordering on the men where the most popular men (resp., the most popular women) are ranked first, we follow a reasoning similar to the one considered in $[15,17]$, that is, we apply a voting rule [1] to the preferences given by the women (resp., by the men). More precisely, such a voting rule takes in input the preference values given by the women over the men (resp., given by the men over the women) and returns a strict total order over the men (resp., women). 
Definition 3 (lex-male-optimal). Consider an SMW P, a natural number $\alpha$, and a voting rule $r$. Let us denote with $o_{m}$ (resp., $o_{w}$ ) the strict total order over the men (resp., over the women) computed by applying $r$ to the preference values that the women give to the men (resp., the men give to the women). An $\alpha$-stable marriage $M$ is lex-male-optimal w.r.t. $o_{m}$ and $o_{w}$, if, for every other $\alpha$-stable marriage $M^{\prime}$, the following conditions hold:

- there is a man $m_{i}$ such that $M\left(m_{i}\right) \succ_{o_{w}} M^{\prime}\left(m_{i}\right)$,

- for every man $m_{j} \prec_{o_{m}} m_{i}, M\left(m_{j}\right)=M^{\prime}\left(m_{j}\right)$.

Proposition 2. Given an $S M W P$, a strict total ordering $o_{m}$ (resp., ow over the men (resp., women),

- there is a unique lex-male-optimal $\alpha$-stable marriage w.r.t. $o_{m}$ and $o_{w}$, say $L$.

- L may be different from the male-optimal stable marriage of $c(P)$;

- if $\alpha(P)$ has a unique undominated weakly stable marriage, say $L^{\prime}$, then $L$ coincides with $L^{\prime}$, otherwise $L$ is one of the undominated weakly stable marriages of $\alpha(P)$.

Example 7. Let us consider the SMW, $P$, shown in Example 5. We have shown previously that this problem has two $\alpha$-weakly stable marriages that are undominated. We now want to discriminate among them by considering the lex-maleoptimality notion. Let us consider as voting rule the rule that takes in input the preference values given by the women over the men (resp., by the men over the women) and returns a strict preference ordering over the men (resp., women). This preference ordering is induced by the overall score that each man (resp., woman) receives: men (women) that receive higher overall scores are more preferred. The overall score of a man $m$ (resp., woman $w$ ), say $s(m)$ (resp., $s(w)$ ), is computed by summing all the preference values that the women give to him (the men give to her). If two candidates receive the same overall score, we use a tie-breaking rule to order them. If we apply this voting rule to the preference values given by the women in $P$, then we obtain

$$
\begin{aligned}
& -s\left(m_{1}\right)=8+3=11, \\
& -s\left(m_{2}\right)=5+1=6,
\end{aligned}
$$

and thus the ordering $o_{m}$ is such that $m_{1} \succ_{o_{m}} m_{2}$. If we apply the same voting rule to the preference values given by the men in $P$,

$$
\begin{aligned}
& -s\left(w_{1}\right)=3+4=7, \\
& -s\left(w_{2}\right)=2+2=4,
\end{aligned}
$$

and thus the ordering $o_{w}$ is such that $w_{1} \succ_{o_{w}} w_{2}$. The lex-male-optimal $\alpha$-stable marriage w.r.t. $o_{m}$ and $o_{w}$ is the marriage $M_{1}=\left\{\left(m_{1}, w_{1}\right),\left(m_{2}, w_{2}\right)\right\}$. 


\subsection{Finding the lex-male-optimal $\alpha$-stable marriage}

It is possible to find optimal $\alpha$-stable marriages by adapting the GS-algorithm for classical stable marriage problems [4].

Given an SMW $P$ and a natural number $\alpha$, by Theorem 1 , to find an $\alpha$-stable marriage it is sufficient to find a weakly stable marriage of $\alpha(P)$. This can be done by applying the GS algorithm to any linearization of $\alpha(P)$.

Given an SMW $P$, a natural number $\alpha$, and two orderings $o_{m}$ and $o_{w}$ over men and women computed by applying a voting rule to $P$ as described in Definition 3 , it is possible to find the $\alpha$-stable marriage that is lex-male-optimal w.r.t $o_{m}$ and $o_{w}$ by applying the GS algorithm to the linearization of $\alpha(P)$ where we order incomparable pairs, i.e., the pairs that differ for less than $\alpha$ in $P$, in accordance with the orderings $o_{m}$ and $o_{w}$.

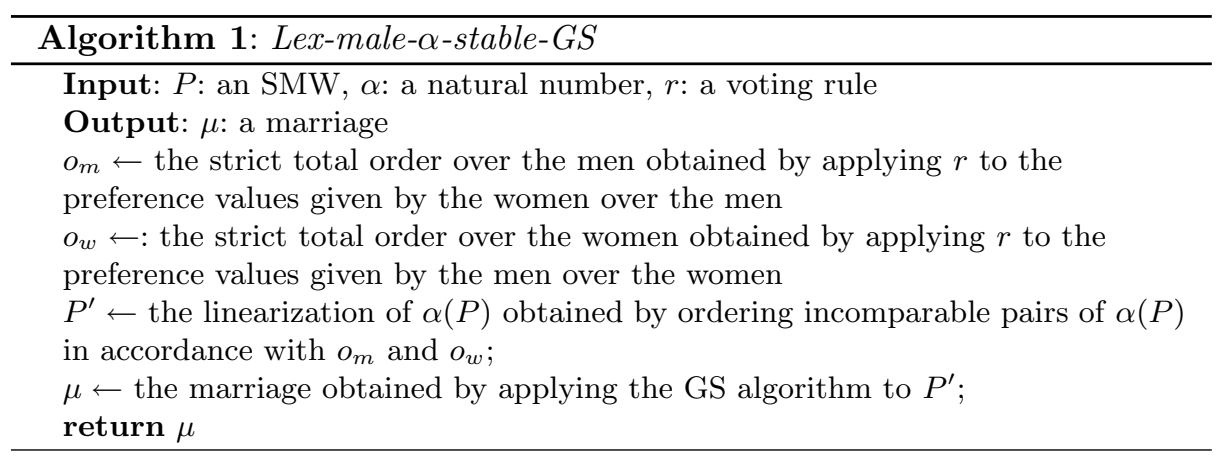

Proposition 3. Given an $S M W P$, a natural number $\alpha, o_{m}$ (resp., $o_{w}$ ) an ordering over the men (resp., women), algorithm Lex-male- $\alpha$-stable-GS returns the lex-male-optimal $\alpha$-stable marriage of $P$ w.r.t. $o_{m}$ and $o_{w}$.

\section{Stability notions relying on links}

Until now we have generalized the classical notion of stability by considering separately the preferences of the men and the preferences of the women. We now intend to define new notions of stability that take into account simultaneously the preferences of the men and the women. Such a new notion will depend on the strength of the link of the married people, i.e., how much a man and a woman want to be married with each other. This is useful to obtain a new notion of stable marriage, that looks at the happiness of the pairs (man,woman) rather than at the happiness of the members of a single sex.

A way to define the strength of the link of two people is the following.

Definition 4 (link additive-strength). Given a man $m$ and a woman $w$, the link additive-strength of the pair $(m, w)$, denoted by $l a(m, w)$, is the value 
obtained by summing the preference value that $m$ gives to $w$ and the preference value that $w$ gives to $m$, i.e., la $(m, w)=p(m, w)+p(w, m)$. Given a marriage $M$, the additive-link of $M$, denoted by $l a(M)$, is the sum of the links of all its pairs, i.e., $\sum_{\{(m, w) \in M\}} l a(m, w)$.

Notice that we can use other operators beside the sum to define the link strength, such as, for example, the maximum or the product.

We now give a notion of stability that exploit the definition of the link additive-strength given above.

Definition 5 (link-additive-stability). Given a marriage $M$, a man $m$, and a woman $w$, the pair $(m, w)$ is a link-additive-blocking pair for $M$ if the following conditions hold:

$-l a(m, w)>l a\left(m^{\prime}, w\right)$,

$-l a(m, w)>l a\left(m, w^{\prime}\right)$,

where $m^{\prime}$ is the partner of $w$ in $M$ and $w^{\prime}$ is the partner of $m$ in $M$. A marriage is link-additive-stable if it does not contain link-additive-blocking pairs.

Example 8. Let $\left\{w_{1}, w_{2}\right\}$ and $\left\{m_{1}, m_{2}\right\}$ be, respectively, the set of women and men. Consider the following instance of an SMW, $P$ :

$-m_{1}: w_{1}^{[30]}>w_{2}^{[3]}$

$-m_{2}: w_{1}^{[4]}>w_{2}^{[3]}$,

$-w_{1}: m_{2}^{[6]}>m_{1}^{[5]}$

$-w_{2}: m_{1}^{[10]}>m_{2}^{[2]}$.

In this example there is a unique link-additive-stable marriage, that is $M_{1}=$ $\left\{\left(m_{1}, w_{1}\right),\left(m_{2}, w_{2}\right)\right\}$, which has additive-link $l a\left(M_{1}\right)=35+5=40$. Notice that such a marriage has an additive-link higher than the male-optimal stable marriage of $c(P)$ that is $M_{2}=\left\{\left(m_{1}, w_{2}\right),\left(m_{2}, w_{1}\right)\right\}$ which has additive-link $l a\left(M_{2}\right)=13+10=23$.

The strength of the link of a pair (man,woman), and thus the notion of link stability, can be also defined by considering the maximum operator instead of the sum operator.

Definition 6 (link maximal-strength). Given a man $m$ and a woman $w$, the link maximal-strength of the pair $(m, w)$, denoted by $\operatorname{lm}(m, w)$, is the value obtained by taking the maximum between the preference value that $m$ gives to $w$ and the preference value that $w$ gives to $m$, i.e., $\operatorname{lm}(m, w)=\max (p(m, w), p(w, m))$. Given a marriage $M$, the maximal-link of $M$, denoted by $\operatorname{lm}(M)$, is the maximum of the links of all its pairs, i.e., $\max _{\{(m, w) \in M\}} \operatorname{lm}(m, w)$.

Definition 7 (link-maximal-stability). Given a marriage $M$, a man $m$, and a woman $w$, the pair $(m, w)$ is a link-maximal-blocking pair for $M$ if the following conditions hold: 
$-\operatorname{lm}(m, w)>\operatorname{lm}\left(m^{\prime}, w\right)$

$-\operatorname{lm}(m, w)>\operatorname{lm}\left(m, w^{\prime}\right)$,

where $m^{\prime}$ is the partner of $w$ in $M$ and $w^{\prime}$ is the partner of $m$ in $M$. A marriage is link-maximal-stable if it does not contain link-maximal-blocking pairs.

\subsection{Relations with other stability notions}

Given an SMW $P$, let us denote with $\operatorname{Linka}(P)$ (resp., $\operatorname{Linkm}(P)$ ) the stable marriage problem with ties obtained from $P$ by changing every preference value that a person $x$ gives to a person $y$ with the value $l a(x, y)$ (resp., $\operatorname{lm}(x, y)$ ), by changing the preference rankings accordingly, and by considering only these new preference rankings.

Let us denote with $I_{l a}(P)$ (resp., $I_{l m}(P)$ ) the set of the link-additive-stable marriages (resp., link-maximal-stable marriages) of $P$ and with $I_{w}(\operatorname{Linka}(P))$ (resp., $\left.I_{w}(\operatorname{Linkm}(P))\right)$ the set of the weakly stable marriages of $\operatorname{Linka}(P)$ (resp., $\operatorname{Linkm}(P))$. It is possible to show that these two sets coincide.

Theorem 2. Given an $S M W P, I_{l a}(P)=I_{w}(\operatorname{Linka}(P))$ and $I_{l m}(P)=I_{w}(\operatorname{Linkm}(P))$.

Proof. Let us consider a marriage $M$. We first show that if $M \in I_{w}(\operatorname{Linka}(P))$ then $M \in I_{l a}(P)$. If $M \notin I_{l a}(P)$, there is a pair $(m, w)$ that is a link-additiveblocking pair, i.e., $l a(m, w)>l a\left(m, w^{\prime}\right)$ and $l a(m, w)>l a\left(m^{\prime}, w\right)$, where $w^{\prime}$ (resp., $\left.m^{\prime}\right)$ is the partner of $m$ (resp., $w$ ) in $M$. Since $l a(m, w)>l a\left(m, w^{\prime}\right), m$ prefers $w$ to $w^{\prime}$ in the problem $\operatorname{Link} a(P)$, and, since $l a(m, w)>l a\left(m^{\prime}, w\right), w$ prefers $m$ to $m^{\prime}$ in the problem Linka $(P)$. Hence $(m, w)$ is a blocking pair for the problem $\operatorname{Linka}(P)$. Therefore, $M \notin I_{w}(\operatorname{Linka}(P))$.

We now show that if $M \in I_{l a}(P)$ then $M \in I_{w}(\operatorname{Linka}(P))$. If $M \notin I_{w}(\operatorname{Linka}(P))$, there is a pair $(m, w)$ that is a blocking pair for $I_{w}(\operatorname{Link} a(P))$, i.e., $m$ prefers $w$ to $w^{\prime}$ in the problem $\operatorname{Link} a(P)$, and $w$ prefers $m$ to $m^{\prime}$ in the problem $\operatorname{Link} a(P)$. By definition of the problem $\operatorname{Link} a(P), l a(m, w)>l a\left(m, w^{\prime}\right)$ and $l a(m, w)>l a\left(m^{\prime}, w\right)$. Therefore, $(m, w)$ is a link-additive-blocking pair for the problem $P$. Hence, $M \notin I_{l a}(P)$.

It is possible to show similarly that $I_{l m}(P)=I_{w}(\operatorname{Linkm}(P))$.

When no preference ordering changes in $\operatorname{Linka}(P)($ resp., $\operatorname{Linkm}(P))$ w.r.t. $P$, then the link-additive-stable (resp., link-maximal-stable) marriages of $P$ coincide with the stable marriages of $c(P)$.

Proposition 4. Given an $S M W P$, if $\operatorname{Linka}(P)=c(P)(\operatorname{Linkm}(P)=c(P))$, then $I_{l a}(P)=I(c(P))$ (resp., $\left.I_{l m}(P)=I(c(P))\right)$.

If there are no ties in $\operatorname{Linka}(P)($ resp., $\operatorname{Linkm}(P))$, then there is a unique link-additive-stable marriage (resp., link-maximal-stable marriage) with the highest link.

Proposition 5. Given an $S M W P$, if Linka $(P)$ (resp., Linkm $(P))$ has no ties, then there is a unique link-additive-stable (resp., link-maximal-stable) marriage with the highest link. 
If we consider the definition of link-maximal-stability, it is possible to define a class of SMWs where there is a unique link-maximal-stable marriage with the highest link.

Proposition 6. In an $S M W P$ where the preference values are all different, there is a unique link-maximal-stable marriage with the highest link.

\subsection{Finding link-additive-stable and link-maximal-stable marriages with the highest link}

We now show that for some classes of preferences it is possible to find optimal link-additive-stable marriages and link-maximal-stable marriages of an SMW by adapting algorithm GS, which is usually used to find the male-optimal stable marriage in classical stable marriage problems.

By Proposition 2, we know that the set of the link-additive-stable (resp., linkmaximal-stable) marriages of an SMW $P$ coincides with the set of the weakly stable marriages of the SMP $\operatorname{Linka}(P)$ (resp., $\operatorname{Linkm}(P))$. Therefore, to find a link-additive-stable (resp., link-maximal-stable) marriage, we can simply apply algorithm GS to a linearization of $\operatorname{Linka}(P)($ resp., $\operatorname{Linkm}(P))$.

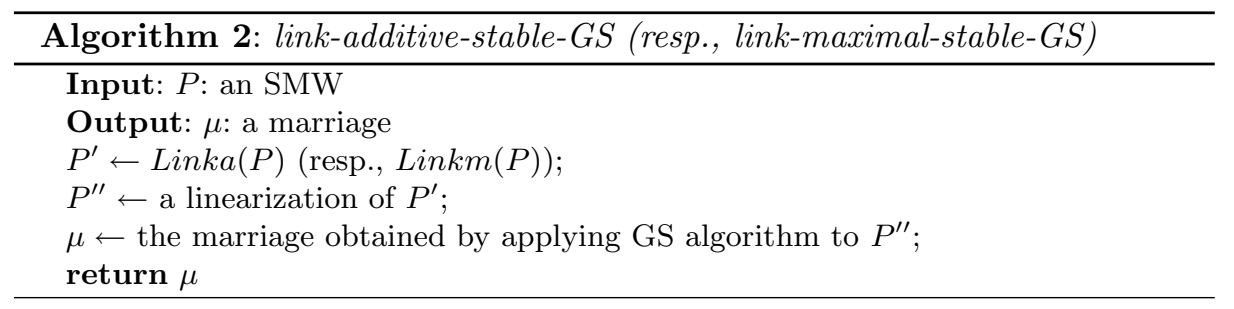

Proposition 7. Given an $S M W P$, the marriage returned by algorithm linkadditive-stable-GS (link-maximal-stable-GS) over $P$, say $M$, is link-additivestable (resp., link-maximal-stable). Moreover, if there are not ties in Linka $(P)$ (resp., Linkm $(P)), M$ is link-additive-stable (resp., link-maximal-stable) and it has the highest link.

When there are no ties in $\operatorname{Linka}(P)$ (resp., $\operatorname{Linkm}(P)$ ), the marriage returned by algorithm link-additive-stable-GS (resp., link-maximal-stable-GS) is male-optimal w.r.t. the profile with links. Such a marriage may be different from the classical male-optimal stable marriage of $c(P)$, since it considers the happiness of the men reordered according to their links with the women, rather than according their single preferences.

This holds, for example, when we assume to have an SMW with preference values that are all different and we consider the notion of link-maximal-stability.

Proposition 8. Given an SMW P where the preference values are all different, the marriage returned by algorithm link-maximal-stable-GS algorithm over $P$ is link-maximal-stable and it has the highest link. 


\section{Manipulation}

In [18] Roth has shown that, when there are at least three men and three women, every stable marriage procedure is manipulable, i.e., there is a profile where an agent, mis-reporting his preferences, obtains a stable marriage which is better than or equal to the one obtained by telling the truth. In stable marriage problems, agents can manipulate in two ways: by changing the preference ordering [18], or by truncating the preference list [5].

We now would like to check if agents have additional ways of manipulating in our context, by changing only the preference weights, while preserving the preference ordering and not truncating the preference list.

In the following, we will call a w-manipulation attempt by an agent $a$ the misreporting of the weights in $a$ 's preferences which preserves the true preference ordering. Also, we will say that a w-manipulation attempt of an agent $a$ is successful if the resulting marriage for $a$ is better than or equal to the marriage obtained by using the true preference weights of $a$, and that a stable marriage procedure is $w$-manipulable if there is a profile with a successful w-manipulation attempt for an agent.

We will show that every stable marriage procedure which returns an $\alpha$-stable marriage, a link-additive, or a link-maximal stable marriage, is w-manipulable.

Theorem 3. Let $\alpha$ be any natural number $>1$. Every procedure which returns an $\alpha$-stable marriage is w-manipulable.

Proof. Let $\left\{w_{1}, w_{2}, w_{3}\right\}$ and $\left\{m_{1}, m_{2}, m_{3}\right\}$ be, respectively, the set of women and men. Consider the following instance of an SMW, say $P,\left\{m_{1}: w_{2}^{[x+2 \alpha]}>\right.$ $w_{1}^{[x+\alpha]}>w_{3}^{[x]}, m_{2}: w_{1}^{[x+2 \alpha]}>w_{2}^{[x+\alpha]}>w_{3}^{[x]}, m_{3}: w_{1}^{[x+2 \alpha]}>w_{2}^{[x+\alpha]}>w_{3}^{[x]}, w_{1}:$ $m_{1}^{[x+\alpha+1]}>m_{2}^{[x+\alpha]}>m_{3}^{[x]}, w_{2}: m_{3}^{[x+2 \alpha]}>m_{1}^{[x+\alpha]}>m_{2}^{[x]}, w_{3}: m_{1}^{[x+2 \alpha]}>$ $\left.m_{2}^{[x+\alpha]}>m_{3}^{[x]}\right\} . P$ has two $\alpha$-stable marriages: $M_{1}=\left\{\left(m_{1}, w_{1}\right),\left(m_{2}, w_{3}\right),\left(m_{3}, w_{2}\right)\right\}$ and $M_{2}=\left\{\left(m_{1}, w_{3}\right),\left(m_{2}, w_{1}\right),\left(m_{3}, w_{2}\right)\right\}$. Assume that $w_{1}$ mis-reports her preferences as follows: $w_{1}: m_{1}^{[x+2 \alpha]}>m_{2}^{[x+\alpha]}>m_{3}^{[x]}$, i.e., assume that she changes the weight given to $m_{1}$ from $x+\alpha+1$ to $x+2 \alpha$. Let us denote with $P^{\prime}$ the resulting problem. $P^{\prime}$ has a unique $\alpha$-stable marriage, that is $M_{1}$, which is the best $\alpha$-stable marriage for $w_{1}$ in $P$. Therefore, it is convenient for $w_{1}$ to change her weights to get a better or equal result w.r.t the one obtained by telling the truth. Also, since $P^{\prime}$ has a unique $\alpha$-stable marriage, every procedure which returns an $\alpha$-stable marriage returns such a marriage. Thus, every procedure is w-manipulable.

Theorem 4. Every stable marriage procedure that returns a link-additive stable marriage is w-manipulable.

Proof. Let $\left\{w_{1}, w_{2}\right\}$ and $\left\{m_{1}, m_{2}\right\}$ be, respectively, the set of women and men. Consider the following instance of an SMW, say $P:\left\{m_{1}: w_{2}^{[6]}>w_{1}^{[4]}, m_{2}\right.$ : $\left.w_{2}^{[5]}>w_{1}^{[2]}, w_{1}: m_{1}^{[3]}>m_{2}^{[2]}, w_{2}: m_{1}^{[5]}>m_{2}^{[2]}\right\} . P$ has a unique link-additive stable marriage, which is $M_{1}=\left\{\left(m_{1}, w_{2}\right),\left(m_{2}, w_{1}\right)\right\}$. Assume that $w_{1}$ mis-reports 
her preferences as follows: $w_{1}: m_{1}^{[100]}>m_{2}^{[2]}$, i.e., she changes the weight given to $m_{1}$ from 3 to 100 . Then, in the new problem, that we call $P^{\prime}$, there is a unique link-additive stable marriage, i.e., $M_{2}=\left\{\left(m_{1}, w_{1}\right),\left(m_{2}, w_{2}\right)\right\}$, which is better for $w_{1}$ in $P$. Since in $P^{\prime}$ there is a unique link-additive-stable marriage, every procedure which returns a link-additive stable marriage will return it. Thus, every procedure is w-manipulable.

A similar result can be shown also for stable marriage procedures that return a link-maximal stable marriage.

Summarizing, even if we don't allow the agents to modify their preference ordering or to truncate their preference list, they can manipulate simply by changing the values of their weights. Moreover, it is possible to see that some ideas to prevent manipulation, such as to assign equal weight to all top alternatives and to put a bound over the relative weights of two consecutive elements in every ordering, are ineffective to avoid w-manipulation.

\section{Conclusions and future work}

In this paper we have considered stable marriage problems with weighted preferences, where both men and women can express a score over the members of the other sex. In particular, we have introduced new stability and optimality notions for such problems and we have compared them with the classical ones for stable marriage problems with totally or partially ordered preferences. Also, we have provided algorithms to find marriages that are optimal and stable according to these new notions by adapting the Gale-Shapley algorithm. Moreover, we have investigated manipulation issues in our context.

We have also considered an optimality notion (that is, lex-male-optimality) that exploits a voting rule to linearize the partial orders. We intend to study if this use of voting rules within stable marriage problems may have other benefits. In particular, we want to investigate if the procedure defined to find such an optimality notion inherits the properties of the voting rule with respect to manipulation: we intend to check whether, if the voting rule is NP-hard to manipulate, then also the procedure on SMW that exploits such a rule is NP-hard to manipulate. This would allow us to transfer several existing results on manipulation complexity, which have been obtained for voting rules, to the context of procedures to solve stable marriage problems with weighted preferences.

Acknowledgments This work has been partially supported by the MIUR PRIN 20089M932N project "Innovative and multi-disciplinary approaches for constraint and preference reasoning".

\section{References}

1. K.J. Arrow, A.K. Sen, and K. Suzumura. Handbook of Social Choice and Welfare. North Holland, Elsevier, 2002. 
2. S. Bistarelli, S. N. Foley, B. O'Sullivan, and F. Santini. From marriages to coalitions: A soft csp approach. In CSCLP, pages 1-15, 2008.

3. S. Bistarelli, U. Montanari, and F. Rossi. Semiring-based constraint solving and optimization. Journal of the ACM, 44(2):201-236, 1997.

4. D. Gale and L. S. Shapley. College admissions and the stability of marriage. Amer. Math. Monthly, 69:9-14, 1962.

5. D. Gale and M. Sotomayor. Semiring-based constraint solving and optimization. American Mathematical Monthly, 92:261-268, 1985.

6. M. Gelain, M. S. Pini, F. Rossi, K. B. Venable, and T. Walsh. Male optimal and unique stable marriages with partially ordered preferences. In Proc. CARE 2009/2010. Springer LNAI 6066, 2010.

7. M. Gelain, M. S. Pini, F. Rossi, K. B. Venable, and T. Walsh. Male optimality and uniqueness in stable marriage problems with partial orders - Extended abstract. In Proc. AAMAS'10, 2010.

8. D. Gusfield. Three fast algorithms for four problems in stable marriage. SIAM J. Comput., 16(1):111-128, 1987.

9. D. Gusfield and R. W. Irving. The Stable Marriage Problem: Structure and Algorithms. MIT Press, Boston, Mass., 1989.

10. M. Halldorsson, R. W. Irving, K. Iwama, D. Manlove, S. Miyazaki, Y. Morita, and S. Scott. Approximability results for stable marriage problems with ties. Theor. Comput. Sci, 306(1-3):431-447, 2003.

11. R. W. Irving. Stable marriage and indifference. Discrete Applied Mathematics, 48:261-272, 1994.

12. R. W. Irving, P. Leather, and D. Gusfield. An efficient algorithm for the "optimal" stable marriage. J. ACM, 34(3):532-543, 1987.

13. J. Liebowitz and J. Simien. Computational efficiencies for multi-agents: a look at a multi-agent system for sailor assignment. Electonic government: an International Journal, 2(4):384-402, 2005.

14. D. Manlove. The structure of stable marriage with indifference. Discrete Applied Mathematics, 122(1-3):167-181, 2002.

15. M. S. Pini, F. Rossi, K. B. Venable, and T. Walsh. Manipulation and gender neutrality in stable marriage procedures. In Proc. $A A M A S^{\prime} 09$, volume 1, pages 665-672, 2009.

16. M. S. Pini, F. Rossi, K. B. Venable, and T. Walsh. Stable marriage problems with quantitative preferences. In Informal Proc. of COMSOC'10 - Third International Workshop on Computational Social Choice, 2010.

17. M. S. Pini, F. Rossi, K. B. Venable, and T. Walsh. Manipulation complexity and gender neutrality in stable marriage procedures. Jornal of Autonomous Agents and Multi-Agent Systems, 22(1):183-199, 2011.

18. A. E. Roth. The economics of matching: Stability and incentives. Mathematics of Operations Research, 7:617-628, 1982.

19. A. E. Roth. The evolution of the labor market for medical interns and residents: a case study in game theory. Journal of Political Economy, 92:991-1016, 1984.

20. A. E. Roth. Deferred acceptance algorithms: History, theory, practice, and open questions. International Journal of Game Theory, Special Issue in Honor of David Gale on his 85th birthday, 36:537-569, 2008.

21. Chung-Piaw Teo, Jay Sethuraman, and Wee-Peng Tan. Gale-shapley stable marriage problem revisited: Strategic issues and applications. Manage. Sci., 47(9):1252-1267, 2001. 